\title{
Lidil
}

Revue de linguistique et de didactique des langues

$63 \mid 2021$

Littératie numérique et didactique des langues et des cultures

\section{De quelques biais cognitifs dans l'enseignement des accents diacritiques en FLE}

Focusing on Cognitive Biases in the Teaching of the Diacritical Accents in French

\section{Marion Dufour}

\section{OpenEdition}

\section{Journals}

Édition électronique

URL : http://journals.openedition.org/lidil/8589

DOI : $10.4000 /$ lidil.8589

ISSN : 1960-6052

Éditeur

UGA Éditions/Université Grenoble Alpes

Édition imprimée

ISBN : 978-2-37747-283-3

ISSN : $1146-6480$

Référence électronique

Marion Dufour, « De quelques biais cognitifs dans l'enseignement des accents diacritiques en FLE », Lidil [En ligne], 63 | 2021, mis en ligne le 30 avril 2021, consulté le 30 avril 2021. URL : http:// journals.openedition.org/lidil/8589; DOI : https://doi.org/10.4000/lidil.8589

Ce document a été généré automatiquement le 30 avril 2021.

(C) Lidil 


\title{
De quelques biais cognitifs dans l'enseignement des accents diacritiques en FLE
}

Focusing on Cognitive Biases in the Teaching of the Diacritical Accents in French

\author{
Marion Dufour
}

\section{Introduction}

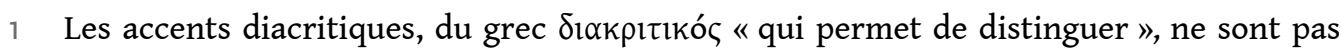
caractéristiques de l'orthographe de toutes les langues. Seules certaines en possèdent. Ils remplissent généralement des fonctions qui varient d'une langue à l'autre: distinguer des homophones, marquer la fin d'une syllabe, renseigner sur une prononciation, rappeler une étymologie, marquer l'accent tonique d'un mot, etc. Le français en possède trois : l'accent aigu, l'accent grave et l'accent circonflexe, qui ont chacun des fonctions particulières ${ }^{1}$. Or, au cours des six années où nous avons enseigné le français dans différentes universités et écoles de commerce à Londres, nous avons constaté que nos étudiants internationaux n'avaient pas une idée claire de la manière d'utiliser les accents diacritiques : certains n'en faisaient aucun usage dans leurs écrits malgré leur niveau parfois avancé, d'autres les utilisaient à mauvais escient parce qu'ils ignoraient le signe diacritique à utiliser (aigu, grave ou circonflexe), les voyelles sur lesquelles il convenait de les mettre; certains autres confondaient des homonymes (comme à préposition et a verbe), d'autres encore les utilisaient comme dans leur langue première. Certes, les nombreuses règles relatives à l'usage de ces accents peuvent en partie expliquer certaines difficultés rencontrées par les apprenants, mais cela n'explique pas tout: la manière dont les enseignants conçoivent la transmission des connaissances, la progression qu'ils mettent en œuvre et la présentation des données qu'ils choisissent doivent également être interrogées. En effet, toute leur habileté réside en leur capacité à sélectionner des savoirs, à les transposer didactiquement (Chevallard, 1991) pour les rendre enseignables - c'est-à-dire adaptés 
à la singularité d'un public donné dans un espace et un temps donnés - pour enfin les transmettre à des fins d'appropriation. Ces nombreuses contraintes conduisent les enseignants à utiliser des heuristiques, c'est-à-dire des «stratégie[s] rapide[s], très efficace[s] - donc économique[s] pour l'enfant - qui marche[nt] très bien, très souvent mais pas toujours [...] » (Houdé, 2019, p. 10) et qui ont pour effet de réduire la réalité, d'en donner une représentation simplifiée voire déformée. Parmi ces heuristiques, on trouve des croyances et des représentations comme des stéréotypes qui nous installent dans des automatismes de pensée et nuisent à notre rationalité. Aussi ces heuristiques peuvent-elles nous entrainer à faire des erreurs de raisonnement ou occasionner des difficultés d'appropriation de certains savoirs. On appelle ces schémas de pensée trompeurs des biais cognitifs (Kahneman \& Tversky, 1974). Les enseignants qui délivrent des savoirs de vive voix en classe et conçoivent des supports pédagogiques sont donc confrontés à une triple obligation : d'abord se montrer réflexifs vis-à-vis des heuristiques qu'ils utilisent pour transmettre, ensuite les présenter comme telles, et non comme des vérités définitives, de manière à ce que leurs apprenants intègrent ces données comme étant relatives à une situation d'enseignement spécifique et contrainte, et enfin entrainer leurs apprenants à résister cognitivement à certaines heuristiques trop rapides qui entrainent des biais de croyance ou de représentativité (clichés, stéréotypes par exemple). Étant donné ces réflexions de départ, et afin de trouver des réponses aux difficultés d'appropriation des accents diacritiques rencontrées par nos apprenants, nous avons examiné comment ces accents étaient présentés dans quatre méthodes de FLE, écrites par des enseignants de FLE et largement utilisées dans le monde du FLE à savoir Latitudes (2008), Alter Ego+(2012), Objectif Express (2013) et Édito (2016). Nous mettons en évidence dans cet article certaines heuristiques problématiques et les biais cognitifs auxquels elles conduisent.

\section{Une introduction des accents confuse et trop succincte}

2 À l'exception d'Edito A1 qui fait complètement l'impasse sur la présentation des signes diacritiques du français, toutes ces méthodes intègrent les accents au sein de leur première unité dans des rubriques aussi diverses qu'inattendues: la rubrique « Retenez » pour Objectif Express 1 (voir p. 17) qui répertorie l'essentiel des énoncés clés à retenir ainsi que le lexique à acquérir pour la réalisation des tâches, la rubrique « phonie-graphie » pour Alter Ego+ A1 (voir p. 190) située en fin du manuel, et le « Précis de grammaire " pour Latitudes 1 (voir p.158), situé également en fin de manuel. À chaque fois, cette introduction a pour but de permettre aux apprenants de remplir la fonction d'épeler les mots en français. Les accents sont présentés en association avec la lettre $e^{2}$ dans des listes où figurent d'autres signes diacritiques (ü, ç), mais aussi l'apostrophe et parfois une consonne redoublée comme c'est le cas dans Objectif Express 1:

Pour épeler :

2. Les caractères spéciaux :

$11:$ deux 1

é : accent aigu

è : accent grave

ê : accent circonflexe [...] (p. 17) 
3 Toutes ces méthodes font le choix d'une introduction très succincte et glottocentrée des accents : nous signifions par ce terme le fait que leurs auteurs ne se préoccupent que de la langue cible. Ce faisant, ils n'invitent pas les apprenants à s'interroger sur leur répertoire langagier en vue d'anticiper les transferts négatifs de traits propres à des langues qu'ils ont déjà acquises vers la langue cible. Or cette démarche s'avèrerait nécessaire. En effet, certaines langues ne possèdent quasiment aucun signe diacritique (cas de l'anglais), ce qui peut amener l'apprenant à penser qu'ils sont accessoires - une de mes apprenantes anglaises les qualifiait de « décoration ». Dans d'autres langues, ces mêmes accents se rapportent parfois à des lettres et à des usages différents de ceux du français. C'est par exemple le cas de l'italien qui ignore l'accent circonflexe, et utilise les accents aigu et grave pour marquer l'accent tonique des mots. En italien, l'accent grave concerne toutes les voyelles $-i$ et $o$ comprises - et sert aussi à noter $e$ et o ouverts, alors que l'accent aigu est utilisé seulement sur les voyelles $e$ et $o$ et note un timbre fermé. Aussi, un apprenant italien ne manquera-t-il pas de s'interroger sur les possibles correspondances avec les accents de l'italien et d'être tenté de transférer ces derniers mécaniquement dans la graphie du français. Cette tendance explique les productions du type il serà sur le modèle de l'italien lui sarà. Le caractère trop succinct de cette introduction constitue donc une heuristique problématique qui ne permet pas aux apprenants d'inhiber les représentations et les automatismes d'écriture qu'ils auront développés dans d'autres langues.

\section{Une catégorisation des accents trompeuse}

4 L'histoire des accents diacritiques du français est intimement liée à celle de la syllabe graphique. En effet, une de leurs fonctions a été de remplacer les lettres muettes qui fermaient les syllabes jusqu'au XvII ${ }^{\mathrm{e}}$ siècle. C'est grâce à leur introduction que feb/urier est devenu février, brief/ue brève, es/cripre écrire, niep/ce nièce, empes/cher empêcher, es/ glise église, etc. (voir Blanche-Benveniste \& Chervel, 1978, p. 66-67). Ceci explique que ces accents apparaissent quasiment toujours en syllabe graphique ouverte $(\mathrm{CV})^{3}$. Le Conseil supérieur de la langue française (1990) énonce ce fait sous la forme suivante : "La lettre $e$ ne reçoit un accent aigu ou grave que si elle est en finale de la syllabe graphique : é/tude, mais es/poir, mé/prise mais mer/cure, inté/ressant mais intel/ligent, etc. » (p. 10) Concernant le choix de l'accent (aigu, grave ou circonflexe) sur la lettre $e$, il dépend le plus souvent de la composition de la syllabe graphique qui suit cet $e$ : si cette syllabe graphique contient un $e$ instable (qui peut être ou ne pas être prononcé) alors le $e$ de la syllabe précédente porte un accent grave ou circonflexe: mère, même, évènement. Dans tous les autres contextes, on utilise l'accent aigu. La compréhension du fonctionnement des accents diacritiques est donc indissociable de l'introduction de la syllabe graphique. Pourtant, il n'existe pas dans ces manuels de rubrique consacrée à l'orthographe à proprement parler, et le découpage syllabique de l'écrit ne fait pas partie de leur programme ${ }^{4}$. Une fois passée la phase d'introduction aux accents décrite plus haut, ces derniers apparaissent toujours au sein de rubriques dont les intitulés annoncent les approches phonographique et phonétique suivant lesquelles ils seront abordés : elles s'intitulent " Phonie-graphie » dans Alter Ego+, « des sons et des lettres » dans Latitudes, ou simplement «Phonétique » dans Édito et «Prononcez » dans Objectif Express. Il résulte de cette approche exclusivement phonographique des accents de nombreux problèmes. 


\subsection{Des règles complexifiées}

D'abord, l'absence de prise en compte des liens qui unissent accents diacritiques et syllabes graphiques conduit à une complexification des règles qui régissent l'utilisation des accents, comme on peut le voir dans deux encadrés de Latitudes 1 ci-dessous. En effet, le premier encadré répertorie les contextes dans lesquels $e$ n'a pas d'accent de la manière suivante :

Le «e » n'a pas d'accent et se prononce $[\varepsilon]$

- devant un « $\mathrm{x}$ » : exemple, exercice, texte

- devant une consonne doublée : elle, lunettes, message

- devant « $\mathrm{s} »$, , $\mathrm{r}$ » et « 1 » + autre consonne : reste, merci, belge. (p. 99)

Or, si les auteurs avaient eu une meilleure connaissance des relations unissant syllabes graphiques et accents diacritiques, ils auraient pu résumer ces trois règles en une seule, à savoir comme nous l'avons induit plus haut, qu'e ne porte pas d'accent en syllabe graphique fermée (CVC) et devant $x$ composé phonétiquement de deux sons consonantiques : $[\mathrm{g}]+[\mathrm{z}]$ (comme dans eg/zema) ou [k]+[s] (comme dans ec/stasy). Un peu plus loin, un second encadré donne la liste des nombreux contextes que l'apprenant devra mémoriser afin «de bien choisir entre l'accent aigu et l'accent grave » (voir le guide pédagogique, 2008, p. 158) :

- é + a, e, é, i, o, u, y : idéal, théâtre

- é + consonne + a, e, é, è, i, o, u, y : départ

Remarque : «é » est souvent la première ou la dernière lettre d'un mot : école, été.

- ̀̀ + consonne (+ e muet) : très, chère

Remarque : « è » n'est jamais la première ou la dernière lettre d'un mot.

- $\hat{\mathbf{e}}$, dans un nombre de mots limité : être, fête, prêt. (p. 109)

Or, encore une fois, les trois premières règles pourraient être simplifiées en une seule, à savoir, comme nous l'avons énoncé plus haut, que l'e accent grave est la plupart du temps concomitant de la présence d'un $e$ instable dans la syllabe qui le suit. Dans tous les autres cas, on utilise un accent aigu. On mesure donc ici à quel point l'introduction de la notion de syllabe, en plus de contribuer à simplifier les règles, est porteuse de sens et permettrait à l'apprenant de construire une représentation cohérente, plutôt qu'arbitraire de l'utilisation des accents.

\subsection{Un biais de conservatisme}

8 Par ailleurs, en favorisant une approche phonographique, c'est-à-dire une approche qui part du son pour aller vers la graphie, les fonctions des accents ne sont pas mises en évidence ; c'est le cas dans l'exercice ci-dessous tiré d'Alter Ego+ A2 (voir le dossier 6, p. 170) où l'apprenant est invité à récapituler dans un tableau toutes les graphies pouvant transcrire le son $[\varepsilon]$, ce qui revient à mettre toutes ces graphies sur le même plan.

a) Écoutez et soulignez tous les mots qui contiennent le son [ع] comme terre. [...]

b) Classez les mots que vous avez soulignés dans le tableau.

\begin{tabular}{|l|l|l|l|l|l|l|l|}
\hline $\begin{array}{l}\grave{e}+\text { cons } \\
+e \text { caduc }\end{array}$ & $\begin{array}{l}e+\text { cons. } \\
\text { prononcée(s) }\end{array}$ & $\begin{array}{l}\text { ai + cons. } \\
\text { prononcée }\end{array}$ & $\begin{array}{l}\text { ai, ầ ou } a i \\
+ \text { lettre muette }\end{array}$ & $\begin{array}{l}\hat{e} \text { (souvent devant } t \\
\text { à la place de } s)^{5}\end{array}$ & $e+x$ & $-e i$ & $\begin{array}{l}-e t /-e c t(e n \\
\text { fin de mot) }\end{array}$ \\
\hline
\end{tabular}




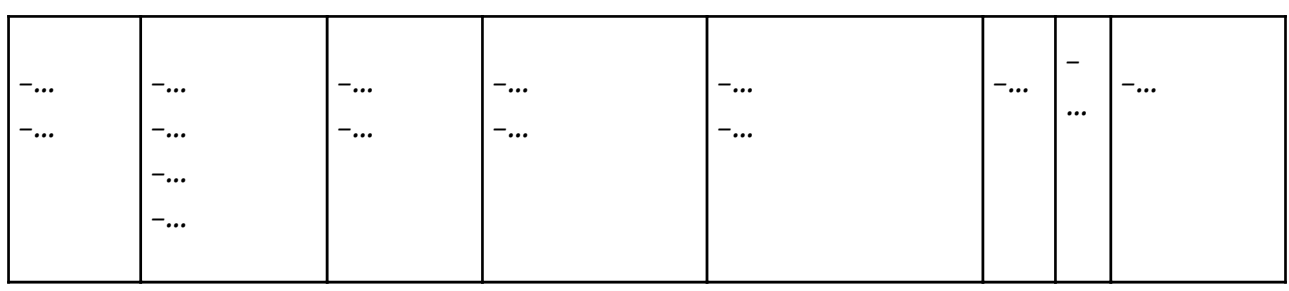

On connait pourtant depuis longtemps les limites d'une telle approche de l'orthographe comme le rappellent Blanche-Benveniste et Chervel (1978) :

On se plaisait jadis à compter le nombre des graphies qui, dans notre écriture, peuvent transcrire un même phonème. L'exercice est vain, car il prend le problème par le mauvais bout. Tout raisonnement qui part du phonème pour aboutir au graphème se termine en imprécations contre l'illogisme du système. Il caractérise assez bien les limites méthodologiques d'un certain réformisme. Il y a pourtant des siècles que la pédagogie a compris qu'on n'enseigne l'écriture française qu'en respectant la direction essentielle, du graphème vers le phonème. (p. 114)

La présence de cet exercice dans cette méthode récente suggère donc un biais cognitif de conservatisme: malgré de nouvelles informations et la démonstration de l'inefficacité de cette approche, les auteurs ne parviennent pas à réviser suffisamment leur croyance.

\subsection{Un biais de corrélation}

11 En outre, cette approche qui associe systématiquement phonie et graphie, est porteuse d'un biais de corrélation visible dans l'exercice de l'unité 6 du cahier d'activités d'Objectif Express 1 (2013, p. 56) ${ }^{6}$ :

Phonie-graphie. 20. Le vocabulaire des transports. Le $e$.

Complétez avec $e, \dot{e}, \grave{e}, \hat{e}, e t, e r$ :

\begin{tabular}{|l|l|l|}
\hline 1. un a...roport & 6. d...coller & 11. une r...servation \\
\hline 2. un all...-retour & 7. un d...part & 12. un si...ge \\
\hline 3. un arr...t de bus & 8. une d...stination & 13. un t...rminal \\
\hline 4. un att...rrissage & 9. des passag...s & 14. un traj... \\
\hline 5. un bill... & 10. la premi...re classe & 15. arriv...e \\
\hline
\end{tabular}

En effet, outre le fait que l'on ait du mal à percevoir la dimension phonétique de cet exercice étant donné qu'il s'appuie uniquement sur la mémoire visuelle des apprenants ayant déjà rencontré ces mots au détour de textes de l'unité 6 , sa consigne donne à penser qu'une graphie renvoie nécessairement à un son, autrement dit qu'il existe des sons inhérents aux graphies $e, e ́, \dot{e}, \hat{e}, e t$, et er, or ce n'est pas le cas. Par exemple, l'accent circonflexe que l'on trouve en 3. arrêt de bus ne renseigne pas sur l'articulation du $e$. Cet accent a été introduit au début du xviII ${ }^{e}$ siècle pour marquer la longueur de la voyelle perçue à l'époque et indiquer la syncope du s d'une ancienne graphie arrest 
(Cerquiglini, 1995, p. 99). Encore une fois, ce biais de corrélation est courant, comme nous le rappellent Blanche-Benveniste et Chervel (1978) :

Il n'est pas de confusion plus fréquemment attestée dans l'histoire de la linguistique que la confusion de la lettre et du son, du graphique et du parler, de l'écriture et de la parole. [...] Incontestablement, ce qui a donné naissance à cet état d'esprit, c'est une pédagogie orientée vers l'écriture et utilisant sans cesse l'écriture. (p. 17-18)

\subsection{Un biais de cadrage}

On pourrait penser que le fait qu'un exercice d'orthographe apparaisse malencontreusement dans une rubrique de phonie-graphie constitue une erreur exceptionnelle. Cependant, on la retrouve aussi dans la troisième unité d'Édito A1 où l'auteure de la rubrique "Phonétique» attire l'attention de l'apprenant sur deux graphies qu'elle juge important qu'il apprenne très tôt à distinguer pour bien lire, à savoir le eaccent aigu et le e sans accent en position finale de mot: "Écoutez et observez les mots. Barrez les lettres finales non prononcées. Qu'est-ce que vous remarquez? a) dimanche b) surgelé c) pâtisserie d) supermarché » (p.52). Dans l'enregistrement qui accompagne cet exercice tous les $e$ finaux sans accent sont muets. Il ne s'agit donc pas d'un exercice de phonétique ni de phonie-graphie. Il s'agit d'une part de sensibiliser l'apprenant au fait qu'une lettre ne correspond pas nécessairement à un son, et d'autre part que l'accent joue un rôle distinctif ; or, le cadre phonétique proposé pour réfléchir au phénomène observé ne permet pas à l'auteure d'aller au-delà de l'heuristique mentionnée dans le titre de la rubrique : « $e$ final [est] non prononcé, éfinal [est] prononcé » (p. 52). Nous identifions donc ici un biais de cadrage que l'on retrouve dans Alter $\mathrm{Ego}^{+} \mathrm{A1}$, dans un exercice proposant une heuristique proche de celle énoncée ci-dessus, à la différence qu'elle concerne cette fois l'accent grave. Cet exercice qui apparait dans la rubrique «Phonie-graphie» du dossier 4 s'intéresse à l'alternance observée dans les radicaux de verbes en -ener, -eler, eter, conjugués au présent de l'indicatif, par exemple: nous nous levons, tu te lèves. Suivant la consigne de cet exercice qui s'accompagne d'un enregistrement, il s'agit de dire si l'on entend dans ces formes verbales, dans lesquelles tous les $e$ internes ont été prononcés, «le son $[\varepsilon]$ comme Irène ou le son [ə] comme Denise » (p. 192). L'exercice propose donc l'heuristique suivante: " $e$ non final se prononce [ə], è non final se prononce $[\varepsilon]$ ». Or, l'auteure de la rubrique omet de mentionner que dans le premier contexte, la lettre $e$ non accentuée peut aussi avoir la valeur zéro. En effet, levons peut se lire aussi bien [ləvõ] que [lvõ], achetez [a〕əte] que [afte]. On réalise donc ici que l'approche phonographique adoptée ne laisse pas de place pour la valeur zéro du $e$, et qu'elle ne permet pas de comprendre le pourquoi du phénomène observé. Pourtant, ce phénomène s'explique et relève en fait moins d'une question phonétique que d'une question prosodique. Il s'explique en effet par la loi d'alternance ou de position ${ }^{7}$ qui fait que l'on prononce différemment le premier $e$ du radical du verbe je me lève constitutif de la syllabe proéminente porteuse de l'accent lexical ${ }^{8}$, de celui de nous nous levons, dont la syllabe n'est pas proéminente puisqu'en français l'accent lexical est toujours positionné sur la dernière syllabe phonique d'un groupe accentuel (Martin, 2009, p. 15). Les formes orthographiques de ces radicaux ne sont donc pas arbitraires mais dues au fait que la syllabe proéminente se trouve à une place différente dans ces deux mots. C'est donc encore un biais de cadrage que l'on observe ici : la consigne de l'exercice invite l'apprenant à se focaliser sur des lettres et des sons alors que le phénomène à 
observer est beaucoup plus large puisqu'il est de l'ordre de la syllabe et de l'accentuation, c'est-à-dire du fonctionnement de la langue.

\subsection{Une croyance répandue}

Enfin, le point commun à toutes ces méthodes est de croire que $e$ accent aigu, $e$ accent grave et $e$ accent circonflexe transcrivent rigoureusement les sons [e] pour l'accent aigu et $[\varepsilon]$ pour les deux autres accents sur $e$ dans tous les contextes. Cette croyance également largement diffusée par le Conseil supérieur de la langue française (1990, p. 10), Le Bon usage (2011, p. 108-109) et le Petit Robert (2017) ${ }^{9}$, s'oppose pourtant à la description phonologique du français contemporain qui montre que le timbre des voyelles en syllabes non finales de mots est « variable et souvent difficile à déterminer avec précision" (Lyche, 2003, p. 351). En effet, la loi de position "est moins transparente pour les voyelles non finales où elle est contrecarrée par d'autres tendances comme l'harmonie vocalique et la fidélité à la racine » (ibid.). C'est également ce que signifie Wioland (1991), en disant que l'opposition de timbres $[e] /[\varepsilon]$ est neutralisée en syllabes phoniques non finales de mots, au profit d'un timbre moyen. Aussi trouve-t-il "pédagogiquement préférable» de transcrire cette neutralisation $\operatorname{par}[E]$. Cette proposition didactique apparait dans la rubrique « Phonétique » d'Édito A2 intitulée "Prononcer e, é, è » (p.150). Cependant, sa mise en œuvre se révèle infructueuse car l'auteure de la rubrique oublie de faire cas du contexte syllabique dans lequel cette neutralisation a lieu. On peut en effet lire dans la première partie intitulée «Échauffement " que "Dans le mot $\mathrm{b}$ [hélicoptère], les $e$ soulignés se prononcent [E] $(=[\mathrm{e}]$ ou $[\varepsilon]) »(\mathrm{p} .150)$ alors que la neutralisation ne concerne que le premier $e$, le second faisant partie de la syllabe phonique finale du mot. On retrouve cette même erreur dans la seconde partie de l'échauffement, où l'apprenant doit établir, parmi un ensemble de mots donnés contenant des e (semaine, père, mère, vert, caractérise, durée, séjour, repos, etc.) la liste des mots dans lesquels un des $e$ se prononce [E], sur le modèle de père, or encore une fois, l'e accent grave de père faisant partie de la syllabe phonique finale de ce mot, la neutralisation n'a donc pas lieu. On retrouve enfin cette erreur dans la règle récapitulant le phénomène observé : "En général, quand la lettre $e$ est à l'intérieur d'un mot $^{10}$ [cherche, éternelle apercevait, fenêtre] et est suivie de deux consonnes, elle se prononce[E]», qui permet de comprendre clairement que son auteure a confondu "à l'intérieur d'un mot » avec « en syllabe phonique non finale de mot », ce qui l'amène à la conclusion erronée que «Les lettres accentuées é, è, ê se prononcent [E] » (p. 150) dans tous les contextes. Paradoxalement, l'auteure du guide pédagogique d'Édito A2 (différente de l'auteure du manuel de l'apprenant), ne revient pas sur la proposition de neutralisation de sa collègue. Au contraire, elle propose des recommandations qui vont à l'encontre de ce phénomène en prescrivant que les é seront prononcés [e] et les $\grave{e}[\varepsilon]$ :

Demander aux apprenants de distinguer le $[e]$ des $[\varepsilon]$ dans les mots de la deuxième colonne :

[e] : caractérise, durée, séjour, éternelles, été

$[\varepsilon]$ : père, mère, vert, fenêtre, apercevait, chẹrche, éternelles (p. 205 du guide pédagogique).

Il semble donc que l'auteure du guide pédagogique ait été déroutée par la nouveauté de la proposition de l'auteure du manuel de l'apprenant, ce qui l'a mise dans l'impossibilité de réviser sa croyance. Ainsi, l'introduction de la neutralisation des $e$ en 
syllabes phoniques non finales de mots, qui au départ visait peut-être à tordre le cou à la croyance très largement admise que é transcrit systématiquement le son [e] et è le son $[\varepsilon]$, se révèle inopérante. Une collaboration plus étroite entre les auteures aurait peut-être pu éviter cette erreur. Cependant, au-delà de cette croyance, on peut supposer que la méconnaissance des relations unissant accents diacritiques et syllabes graphiques a également contribué à cette erreur d'interprétation.

\section{Repenser l'enseignement des accents diacritiques}

16 Ainsi, cette étude montre la nécessité de compléter les approches phonétique et phonographique d'une approche qui tienne compte du fonctionnement orthographique de la langue, afin de redonner du sens aux accents diacritiques. Il en va du développement de la conscience orthographique de l'apprenant et du renforcement de ses rôles de lecteur et de scripteur, puisque au-delà de l'approche phonographique, ces méthodes ne font pas de place à l'orthographe.

\subsection{Introduire les accents en relation avec la syllabe graphique et le e fantôme}

Pour rendre plus pertinente la présentation de ces accents, il faudrait d'abord donner la possibilité aux apprenants de se pencher sur l'orthographe des mots pour les amener à observer que les accents apparaissent quasi systématiquement en syllabe ouverte. Cela nécessiterait en amont un apprentissage du découpage syllabique de l'écrit et de porter une attention particulière aux $e$ que l'on qualifie communément de " $e$ muet " alors qu'ils ne le sont pas toujours. C'est pourquoi nous proposons d'utiliser la formule " $e$ fantôm $e^{11}$ » pour ne pas véhiculer une représentation erronée de cet $e$ susceptible d'être matérialisé dans la lecture. En effet, si nous revenons à la question de la prononciation du e final de mots, présentée antérieurement dans l'exercice d'Édito A1 [ «Écoutez et observez les mots. Barrez les lettres finales non prononcées. Qu'est-ce que vous remarquez? a) dimanche b) surgelé c) pâtisserie d) supermarché. » (p. 52)], et que nous nous intéressons au $e$ final de dimanche, on observera qu'à l'écrit il compte toujours trois syllabes $\mathrm{di} / \mathrm{man} / \mathrm{che}$ alors qu'à l'oral, il sera possible dans certains contextes d'en entendre prononcer trois [di.mã..ə], comme c'est par exemple le cas dans le refrain de la chanson Les roses blanches de Berthe Sylva: "C'est aujourd'hui dimanche, tiens ma jolie maman, voici des roses blanches, toi qui les aimes tant ", mais on pourra dans d'autres contextes n'en compter que deux [di.mõ ]], comme c'est le cas dans la chanson de Michel Berger Un dimanche au bord de l'eau : " un dimanch $e$ au bord de l'eau, dans les rires et les sanglots ». Parler de " $e$ fantôme» prend aussi une dimension particulière dans les exercices où l'accent permet de distinguer certains homographes comme c'est le cas dans la rubrique d'objectif Express 1 de la page 101 et dans la rubrique phonie-graphie de Latitudes 2 de la page 17 qui opposent des formes de verbes du premier groupe au présent et au passé-composé : «je mange/j’ai mangé, je prépare/j'ai préparé, je visite/j'ai visité, je travaille/j'ai travaillé [...] 》 (Objectif Express 1, p. 101). Dans cet exercice, où la fonction morphologique de l'accent aigu (distinguer des temps à l'écrit) est mise en évidence, on passe en quelque sorte d'une image mentale immatérielle du e final, à une image acoustique et matérielle par l'intermédiaire de l'accent qui se comporte comme le révélateur d'un phonème qu'on croyait amuï12. 
Ainsi, s'attarder sur l'observation orthographique des mots et s'intéresser au fonctionnement des $e$ de «a) dimanche b) surgelé c) pâtisserie » (Édito A1, p. 52) permet à l'apprenant de prendre conscience de deux choses fondamentales : d'une part qu'une lettre en français ne transcrit pas nécessairement un son, elle peut remplir d'autres fonctions notamment distinctives (par exemple, dans le mot surgelé le premier e permet de sélectionner la valeur [3] dug et de servir d'anticoagulant pour éviter la lecture [gl]), et d'autre part qu'il résulte de cette non-correspondance systématique entre graphie et phonie, une syllabation différente de l'écrit et de la parole. Comment qualifier ce phénomène? Adoptant une approche graphocentrée, Blanche-Benveniste et Chervel (1978, p. 143) ainsi que Jaffré (2003, p.42) appellent "syllabe fictive» la dernière syllabe graphique d'un mot comportant un $e$, pouvant être suivi d'un $-s$ (marque du nombre et de la seconde personne des verbes) ou de -nt (marque de la troisième personne du pluriel des verbes). Bien que discutable puisque la parole ne connait pas de syllabe fictive et que la syllabe écrite est bien réelle, cette appellation heuristique nous parait commode à utiliser dans le cadre de l'apprentissage de la lecture du français, à la différence que nous préférons parler de "syllabe fantôme » afin de signifier qu'elle n'a rien d'imaginaire, mais résulte de l'histoire de la langue, et peut être la trace d'une ancienne prononciation qui continue à exister au plan interne et cognitif, par l'intermédiaire de la graphie qui la rappelle à notre conscience. Enfin, utiliser les heuristiques "syllabe fantôme » et " $e$ fantôme » a aussi pour intérêt de mettre en exergue, grâce à une appellation évocatrice et imagée, des phénomènes complexes qui posent souvent problème aux apprenants dans leur entrée dans la lecture du français, puisque dans la plupart des langues romanes les graphies e, es, ent et en anglais es et ent à la finale des mots font l'objet d'une articulation. Mettre l'accent sur ces phénomènes peut donc aider l'apprenant à inhiber les automatismes de lecture qu'il a développés dans sa L1 ou dans sa L2 (l'anglais le plus souvent).

\subsection{Vers une approche morphographique des accents diacritiques}

Ensuite, plutôt que de choisir une approche qui parte du son pour aller vers la graphie, comme c'est une nouvelle fois le cas dans cet exercice tiré d'Objectif Express 1: "Le son [e]: A. Écoutez et répétez les phrases. Voici l'agent immobilier b. La cuisine est équipée. c. vous voulez visiter? d. voilà votre clé. e. Vous allez payer le loyer. B. Comment s'écrit le son $[\mathrm{e}]$ ? » (p. 101), il serait bon de privilégier la démarche inverse. Cela permettrait de présenter ces différentes graphies comme des marques morphologiques permettant de discriminer l'infinitif, de l'adjectif ou du participe passé (masculin ou féminin) ou bien encore de la deuxième personne du pluriel de la majorité des verbes à l'indicatif présent. Là encore, une démarche de segmentation syllabique pourrait aider l'apprenant à inhiber ses automatismes, en le conduisant à catégoriser ces graphies dans différents paradigmes grammaticaux, en lui fournissant une explication qui ait du sens et en l'alertant sur le risque d'erreur. En outre, ce type d'activité serait l'occasion d'attirer l'attention de l'apprenant sur le fait que « les préfixes dé-, pré- et télé (ex. : dégeler, prévenir, télémesure) » échappent à la règle stipulant qu'un $e$ qui précède une syllabe fantôme porte un accent grave (voir Constant, 2009, p. 21). 


\subsection{Vers une approche lexicographique des accents diacritiques}

Enfin, partir de l'observation de la graphie permettrait aussi d'opérer des rapprochements entre mots d'une même famille de langues. On pourrait par exemple montrer aux apprenants que le choix des consonnes auxiliaires non prononcées en français ${ }^{13}$, ayant pour fonction de sélectionner la valeur sonore de $e$ en finale de mots, à savoir $t, d, z, f$ (uniquement dans clef) et $r^{14}$ : décret, pied, nez, barbier (exceptions : amer, cancer, laser, hiver, enfer, revolver...), est en rapport avec la formation de leurs dérivés en français (décréter, pédestre, barbière). Quant au choix des graphies é et $\hat{e}$, il serait intéressant de montrer que l'on peut établir des correspondances ${ }^{15}$ entre certains radicaux et leurs dérivés en français, mais aussi dans d'autres langues romanes ainsi qu'en anglais (voir les exemples dans les tableaux qui suivent).

\begin{tabular}{|l|l|l|l|l|l|l|l|l|}
\hline \multicolumn{6}{|c|}{ Correspondance entre é, es + consonne en français et $s, e s+$ consonne dans d'autres langues } \\
\hline & $\begin{array}{l}\text { Français } \\
\text { base }\end{array}$ & $\begin{array}{l}\text { Français } \\
\text { dérivé }\end{array}$ & Italien & Catalan & Portugais & Roumain & Espagnol & Anglais \\
\hline \multirow{2}{*}{ p. 47} & école & scolaire & scuola & escola & escola & şcoală & escuela & school \\
\cline { 2 - 8 } & esprit & spirituel & spirito & esperit & espirito & spirit & espíritu & spirit \\
\hline
\end{tabular}

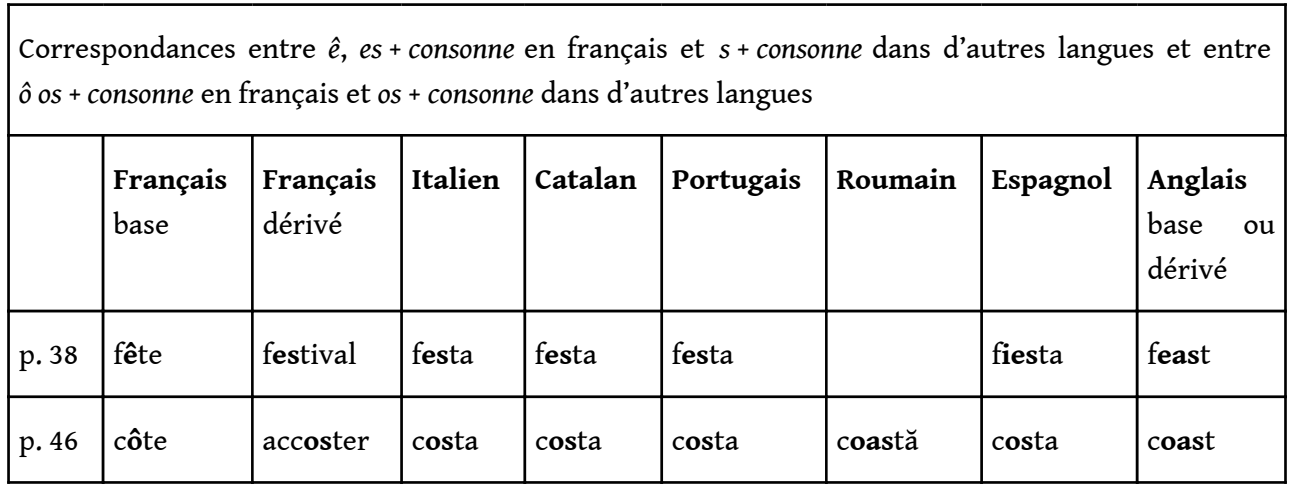

Ces langues pouvant être connues des apprenants, leurs rapprochements leur permettraient de développer en même temps que leur vocabulaire une conscience interlinguistique et de valoriser leur répertoire langagier. Ces exemples montrent donc l'intérêt d'aborder la question des accents diacritiques dans une approche comparative des langues afin d'amener les apprenants à puiser dans les ressources de leur propre répertoire et faciliter la compréhension du fonctionnement de la langue cible.

\section{Conclusion}

21 Il ressort de l'analyse des activités de ces quatre méthodes se rapportant aux accents diacritiques plusieurs biais cognitifs qui font obstacle à leur transposition didactique. Le fait que ces biais soient observés dans toutes ces méthodes alors que leur édition s'étale sur 10 ans de 2006 (pour Alter Ego A1, Alter Ego+ étant une réédition améliorée) à 2016 (pour Edito $A 1$ et A2), pose la question de la transmission de ces biais à leurs 
enseignants-auteurs. Cette étude interroge donc leur culture langagière, c'est-à-dire le « rapport spécifique à la parole et au langage qu'une culture donnée construit comme une donnée partageable dans une communauté » (Siouffi, 2007, p. 268). La présence de ces biais suggère que les enseignants ont peu conscience de la dimension heuristique des savoirs qu'ils transmettent. En témoigne le fait qu'ils n'hésitent pas à formuler certaines heuristiques sous forme de règles sans $\mathrm{y}$ adjoindre de précaution, des règles qui finissent par se transformer en croyances qui rendent ensuite difficile la mise à jour des connaissances et l'intégration de nouvelles données. Aussi nous parait-il important de sensibiliser les enseignants de langues aux sujets des heuristiques et des biais cognitifs afin qu'ils puissent non seulement développer une attitude plus réflexive visà-vis des formes de savoir qu'ils transmettent, mais aussi être en mesure de diagnostiquer et d'anticiper les blocages de leurs apprenants. Dans cette perspective, enseigner dans une approche comparative et contrastive des langues apparait essentiel: se confronter à d'autres systèmes linguistiques permet non seulement d'enrichir ses connaissances et de questionner ses représentations, mais aussi de prendre en compte les savoirs des apprenants sur les points de langue enseignés. Cela permettrait ainsi à l'enseignant d'une part de ne pas céder à une présentation trop simpliste des phénomènes langagiers, et, d'autre part, de comprendre la raison de certaines erreurs récurrentes de ses apprenants et de les anticiper. Il semble en effet que dans certains cas, l'apprentissage de l'inhibition d'un biais sous la forme d'alertes sur le risque d'erreur et la nature du piège perceptif à éviter se révèle beaucoup plus efficace à la réussite d'une tâche que l'explication logique du raisonnement ou la simple répétition de la tâche (voir Houdé, 2019, p. 66). Par ailleurs, pour aider les enseignants à prendre du recul sur les heuristiques qu'ils utilisent, il serait bon d'encourager l'écriture à quatre mains de rubriques pédagogiques, notamment au sein des méthodes de langues, puisqu'on a vu que la multiplicité des auteurs d'une méthode n'impliquait pas nécessairement leur étroite collaboration. Enfin, il conviendrait de multiplier les espaces de formation avec des professionnels en psychologie cognitive, afin que les enseignants soient plus à même d'aider leurs apprenants à développer des stratégies de " résistance cognitive " (Houdé, 2019, p. 70). Ainsi, les enseignants seraient sans doute mieux armés pour œuvrer à une didactisation des savoirs qui conduise plus surement à leur appropriation.

\section{BIBLIOGRAPHIE}

BlanChe-Benveniste, Claire \& CHERVel, André. (1978). L'Orthographe (1 ${ }^{\text {re }}$ éd. 1968). Maspéro.

CATACH, Nina. (1991). L'orthographe en débat. Dossier pour un changement. Nathan Université.

CERqUiglini, Bernard. (1995). L'accent du souvenir. Les Éditions de Minuit.

CHEVAlLARD, Yves. (1991). La transposition didactique : du savoir savant au savoir enseigné. Éditions La pensée sauvage. 
CONSEIL SUPÉRIEUR DE LA LANGUE FRANÇAISE. (1990). Les rectifications de l'orthographe. Journal officiel de la République française, 100, 3-18.

CONSTANT, Chantal. (2009). Grand vadémécum de l'orthographe recommandée. Cinq millepattes sur un nénufar. De Champlain S. F.

GREVISSE, Maurice \& Goose, André. (2011). Le Bon usage. Grammaire langue française (1 ${ }^{\text {re }}$ éd. 1936). De Boeck-Duculot.

Hemming, Erik, Klein, Horst G. \& ReIsSner, Christina. (2011). English - the Bridge to the Romance Languages. Shaker Verlag.

HoudÉ, Olivier. (2019). Apprendre à résister. Pour l'école, contre la terreur (1 $1^{\mathrm{re}}$ éd. 2014). Le PommierHumensis.

JAFFRÉ, Jean-Pierre. (2003). La linguistique et la lecture-écriture : de la conscience phonologique à la variable « orthographe ». Revue des sciences de l'éducation, 29(1), 37-49. <https://doi.org/ 10.7202/009491ar>.

KAHNEMAN, Daniel \& TVERSKY, Amos. (1974). Judgment under Uncertainty: Heuristics and Biases. Sciences, 185(4157), 1124-1131.

LyCHE, Chantal. (2003). La loi de position et le français de Grenoble. Dans E. Delais-Roussaire \& J. Durand (dir.), Corpus et variation en phonologie du français : méthodes et analyses (p. 349-371). Presses universitaires du Mirail.

MARTIN, Philippe. (2009). Intonation du français. Armand Colin.

REY, Alain (dir.). (2017). Le Petit Robert de la langue française (44e édition). Le Robert.

SioufFI, Gilles. (2007). Du sentiment de la langue aux arts du langage. Ela - Études de linguistique appliquée, 147, 265-276. <https://doi.org/10.3917/ela.147.0265>.

WiolAND, François. (1991). Prononcer les mots du français. Hachette.

\section{Méthodes de français et guides pédagogiques cités}

Alcraz, Marion, Braud, Céline, Calvez, Aurélien, Cornuau, Guillaume, Jacob Anne, Pinson, Cécile \& VIDAL, Sandrine. (2016). Édito A1. Méthode de français. Didier.

BRAUD, Céline. (2016). Édito niveau A1. Guide pédagogique. Didier.

DuBoIs, Anne-Lyse \& TAUZIN, Béatrice. (2013). Objectif Express 1. Le monde professionnel en français nouvelle édition. Hachette FLE.

DuBoIs, Anne-Lyse \& TAUZIN, Béatrice. (2013). Objectif Express 1. Le monde professionnel en français nouvelle édition. Cahier d'activités. Hachette FLE.

Heu, Elodie, Abu-Samra, Myriam, Braud, Céline, Brunelle, Michèle, Perrard, Marion \& Pinson, Cécile. (2016). Édito. Méthode de français A2. Didier.

Huck HoAReAu, Saraly, MéRIeux, Régine \& Loiseau, Yves. (2008). Latitudes 1. Méthode de français A1/ A2. Guide pédagogique. Didier.

Hugot, Catherine, Kizirian, Véronique M., WAENDENDries, Monique, Berthet Annie \& Daill, Emmanuelle. (2012). Alter Ego+ A1. Méthode de français. Hachette FLE. 
Hugot, Catherine, KiziRian, Véronique M., WAENDENDRIEs, Monique, BERTHET, Annie, DAILL, Emmanuelle \& SAMPSONIS Béatrix. (2012). Alter Ego+ A2. Méthode de français. Hachette FLE.

MÉRIEUX, Régine \& LoISEAU, Yves. (2008). Latitudes 1. Méthode de français A1/A2. Didier.

MÉRIEUX, Régine, LAINÉ, Emmanuel \& LoISEAU, Yves. (2009). Latitudes 2. Méthode de français A2/B1. Didier.

PERRARD, Marion. (2016). Édito niveau A2 - Guide pédagogique. Didier.

\section{NOTES}

1. Quoique certains accents circonflexes ne jouent actuellement aucun rôle, comme c'est le cas sur câlin ou grâce par exemple, ce qui constitue une indéniable difficulté dans l'apprentissage de l'orthographe du français. Pour de plus amples informations, voir Catach (1991, p. 123-124).

2. Seuls les auteurs de Latitudes 1 prennent le soin de mentionner les différentes lettres auxquelles chacun de ces accents peut être associé : «l'accent grave (è - à - ù) : père $\rightarrow$ P-E accent grave-R-E » (p. 158).

3. Sauf dans quelques mots ayant la finale -êt : arrêt, forêt, intérêt, etc. (voir Grevisse \& Goosse, 2011, p. 111) ou en -ès : très, progrès, succès, etc.

4. Pour prendre connaissance des règles qui régissent le découpage syllabique de la chaine écrite en français, voir Grevisse \& Goosse (2011, p. 37).

5. Notons que c'est l'accent circonflexe qui remplace le $s$ et non $\hat{e}$.

6. On retrouve le même type d'exercice dans Alter Ego+ A2, p. 170, exercice c).

7. La loi de position est généralement formulée comme suit: dans une syllabe phonique fermée $[\mathrm{CVC}]$ la voyelle est ouverte. Dans une syllabe phonique ouverte [CV] la voyelle est fermée.

8. «En français [...] l'accent lexical, participe à la définition du groupe accentuel et se place sur la dernière syllabe (prononcée) de ce groupe » qui « est formé d'une unité de classe ouverte (nom, adjectif, verbe ou adverbe) autour duquel gravitent (éventuellement) des éléments de classe fermée qui sont avec cette unité dans une relation de dépendance (Martin, 1975a)» (Martin, 2009, p. 14-15).

9. Le Petit Robert (2017) transcrit systématiquement les é par des [e] phonétiques.

10. C'est nous qui soulignons.

11. Cette appellation nous a été inspirée par le phénomène connu en musique jazz sous le nom de « note fantôme » : il s'agit d'une note sous-entendue, peu appuyée, voire non jouée, mais que l'on entend quand même inconsciemment (car elle est attachée à une harmonique) et qui donne un effet rythmique particulier à la phrase musicale.

12. «En anc. fr., e était toujours prononcé et, semble-t-il, avec un timbre assez proche de [e] ou de $[\varepsilon]$. C'est en moyen fr. qu'il s'amuït après voyelle, et au XVII ${ }^{e} s$. après consonne. - Les linguistes discutent sur le point de savoir si c'est encore un phonème ou non. » (Grevisse \& Goosse, 2011, p. 39)

13. Les consonnes finales $t, c, r, f, l$ sont toujours prononcées devant $e$ dans les monosyllabes : net, sec, mer, nef, sel. Exceptions : clef, et, pet, il met.

14. Nous n'abordons pas le cas du $s$ car il n'est utilisé comme lettre auxiliaire que dans les monosyllabes : es, ces, des, les, mes, ses, tes.

15. Les exemples de correspondances de ces tableaux sont empruntés à Hemming, Klein et Reissner (2011, p. 28, 38, 46, 47). 


\section{RÉSUMÉS}

$\mathrm{Au}$ cours de six années passées à enseigner le français dans différents universités et écoles de commerce de Londres, nous avons constaté que la plupart de nos apprenants internationaux, parfois de niveau avancé, n'avaient généralement pas une idée claire de la manière d'utiliser les accents diacritiques en français. Cette observation nous a conduite à examiner comment ces accents étaient présentés et enseignés dans quatre méthodes de FLE familières de nos apprenants et communément utilisées dans le monde du FLE (Alter Ego+, Latitudes, Édito et Objectif Express). Notre analyse des activités pédagogiques relatives à l'usage des accents diacritiques nous a permis de mettre en évidence un ensemble de biais cognitifs qui nuisent à leur appropriation et font obstacle à une transposition didactique adéquate des savoirs savants produits sur ces accents.

Throughout my six-year experience of teaching French in different universities and business schools in London, I noticed that most of my international students, sometimes reaching an advanced level, had generally not a clear idea about using diacritical accents in French. This led me to examine how these accents are introduced and taught in four French method books (Alter Ego+, Latitudes, Édito and Objectif Express) familiar to my students and widely used in the FFL world. It emerges from the analysis of the learning activities related to the French diacritical accents a set of cognitive biases that undermine their internalisation and hinder an adequate didactic transposition of the knowledge produced on the French diacritical accents.

\section{INDEX}

Mots-clés : transposition didactique, accents diacritiques, syllabe graphique, heuristiques, biais cognitifs

Keywords : didactic transposition, diacritical accents, graphic syllable, heuristics, cognitive bias

\section{AUTEUR}

\section{MARION DUFOUR}

Professeure invitée à l'Université fédérale du Rio Grande do Sul (UFRGS), Institut de lettres, Département de langues modernes. 